(C) The Authors 2017. This is an Open Access article, distributed under the terms of the Creative

Commons Attribution licence (http://creativecommons.org/licenses/by/4.0/), which permits unrestricted

re-use, distribution, and reproduction in any medium, provided the original work is properly cited.

\title{
Expression profiling indicating low selenium-sensitive microRNA levels linked to cell cycle and cell stress response pathways in the $\mathrm{CaCo}-2$ cell line
}

\author{
Mark J. McCann ${ }^{1 *}$, Kunjana Rotjanapun ${ }^{2}$, John E. Hesketh ${ }^{3}$ and Nicole C. Roy ${ }^{1,4}$ \\ ${ }^{1}$ Food Nutrition \& Health Team, AgResearch Ltd, Grasslands Research Centre, Palmerston North 4442, New Zealand \\ ${ }^{2}$ Department of Food and Nutritional Sciences, University of Reading, PO Box 226, Whiteknights, Reading RG6 6AP, UK \\ ${ }^{3}$ Institute for Cell and Molecular Biosciences, Newcastle University, Newcastle Upon Tyne, NE2 4HH, UK \\ ${ }^{4}$ The Riddet Institute, Massey University, Palmerston North 4442, New Zealand \\ (Submitted 8 December 2016 - Final revision received 14 March 2017 - Accepted 11 April 2017-First published online 2 June 2017)
}

\section{Abstract}

Se is an essential micronutrient for human health, and fluctuations in Se levels and the potential cellular dysfunction associated with it may increase the risk for disease. Although Se has been shown to influence several biological pathways important in health, little is known about the effect of Se on the expression of microRNA (miRNA) molecules regulating these pathways. To explore the potential role of Se-sensitive miRNA in regulating pathways linked with colon cancer, we profiled the expression of 800 miRNA in the CaCo-2 human adenocarcinoma cell line in response to a low-Se $(72 \mathrm{~h}$ at $<40 \mathrm{~nm})$ environment using nCounter direct quantification. These data were then examined using a range of in silico databases to identify experimentally validated miRNA-mRNA interactions and the biological pathways involved. We identified ten Se-sensitive miRNA (hsa-miR-93-5p, hsa-miR-106a-5p, hsa-miR-205-5p, hsa-miR-200c-3p, hsa-miR-99b-5p, hsa-miR-302d-3p, hsa-miR-373-3p, hsa-miR-483-3p, hsa-miR-512-5p and hsa-miR-4454), which regulate $3588 \mathrm{mRNA}$ in key pathways such as the cell cycle, the cellular response to stress, and the canonical Wnt/ $\beta$-catenin, p53 and ERK/MAPK signalling pathways. Our data show that the effects of low Se on biological pathways may, in part, be due to these ten Se-sensitive miRNA. Dysregulation of the cell cycle and of the stress response pathways due to low Se may influence key genes involved in carcinogenesis.

\section{Key words: Selenium: MicroRNA: CaCo-2 cell line: NanoString}

Se is an essential micronutrient for human health ${ }^{(1,2)}$. It is incorporated as selenocysteine into approximately twenty-five Se-containing enzymes (selenoproteins), which have been proposed to have fundamental roles in oxidative defence pathways, thyroid hormone production, inflammation and cardiovascular health ${ }^{(3)}$. Se status has also been linked to cancer risk on the basis of cell, animal and epidemiological studies ${ }^{(4-19)}$, but clinical studies involving Se supplementation have been inconclusive ${ }^{(20)}$. This reflects our incomplete understanding of the mechanisms by which selenoproteins function in complex interdependent cellular pathways, how these are regulated, the effect of SNP in Se-sensitive genes, which may alter how an individual responds to fluctuations in Se status, and the differences in experimental design and approaches. Inadequate Se and the potential cellular dysfunction associated with it may increase the risk for disease ${ }^{(5,7)}$. Severe Se deficiency is rare but sub-optimal intake has been suggested to be prevalent in many parts of the world, and the consequences of inadequate Se status in existing diseases and/or the risk for developing disease further is not well understood ${ }^{(5,7)}$.

MicroRNA (miRNA) are small non-coding RNA, consisting of nineteen to twenty-five nucleotides, which function as regulators of gene expression by affecting translation of mRNA into proteins ${ }^{(21-25)}$. It has been shown that miRNA are critical for the appropriate regulation of fundamental cellular pathways and as such have been implicated in a range of processes that if incorrectly regulated can lead to diseases such as cancer ${ }^{(2,5,7)}$. Furthermore, dietary factors are thought to influence miRNA levels and potentially activate abnormal cellular pathways in cancer development ${ }^{(26-31)}$, and Se status has been shown to affect miRNA levels in the CaCo-2 cell line ${ }^{(32)}$. However, the effect of dysregulated miRNA expression, due to a low-Se environment, on key pathways in cancer development and progression is unclear.

Colon cancer is the third most common cancer in men and the second most common in women globally ${ }^{(33)}$. The disease is characterised by distinct mutations throughout life in key biological cellular functions such as the Wnt/ $\beta$-catenin, $\mathrm{p} 53$, ERK/MAPK and the DNA mismatch repair pathways ${ }^{(34-39)}$. Several components of these canonical pathways have been linked with abnormal miRNA activity ${ }^{(40,41)}$ and Se status ${ }^{(42-51)}$.

We hypothesised that there are miRNA whose expression is dysregulated in a low-Se environment, and that this dysregulation

Abbreviations: miRNA, microRNA; qPCR, quantitative PCR.

*Corresponding author: M. J. McCann, email mark.mccann@agresearch.co.nz 
explains in part the effects of Se status on key biological pathways. To investigate this hypothesis, we used cultured CaCo-2 cells in a low-Se environment for $72 \mathrm{~h}$ before supplementation with or without $40 \mathrm{~nm}$ Se (as sodium selenite). The concentration of Se used ( $40 \mathrm{~nm}$ ) represents a concentration comparable with plasma levels in people with optimal Se status. It has been previously shown that $40 \mathrm{~nm}$ is adequate to maximise expression of the Se-sensitive selenoprotein GPX1 ${ }^{(18,52)}$. The absolute counts of 800 human miRNA were measured using NanoString technology to characterise the miRNA profile in response to a low-Se environment $(<40 \mathrm{~nm})$ compared with a 40-nм Se supplementation. These miRNA data were then assessed in silico, only on the basis of experimentally validated miRNA-mRNA interactions for their effects on biological pathways based on the gene ontology and Reactome databases. The intent of this study was to discover which miRNA were sensitive to low Se so as to provide a robust data set for further functional validation.

\section{Methods}

\section{Cell culture and chemicals}

Authenticated CaCo-2 human colon adenocarcinoma cells were purchased from the American Type Culture Collection. All cell culture reagents and chemicals were purchased from Life Technologies unless otherwise stated. The cells were cultured before the experiment in Dulbecco's Modified Eagle's Medium (DMEM; Sigma-Aldrich), supplemented with $1 \%$ non-essential amino acids, $1 \%$ penicillin-streptomycin solution and $10 \%$ fetal bovine serum, and were maintained at $37^{\circ} \mathrm{C}$ with $5 \% \mathrm{CO}_{2}$ in a humidified atmosphere. A low-Se medium was prepared containing only DMEM, supplemented with $1 \%$ penicillinstreptomycin solution, $5 \mu \mathrm{g} / \mathrm{ml}$ of insulin (Sigma-Aldrich) and $5 \mu \mathrm{g} / \mathrm{ml}$ of transferrin (Sigma-Aldrich).

The effect of Se supplementation in undifferentiated CaCo-2 cells was determined in three biologically independent assays. For each experiment, $5 \times 10^{5} \mathrm{CaCo}-2$ cells were seeded into three wells of a six-well tissue-culture plate (Corning) and cultured at $37^{\circ} \mathrm{C}$ with $5 \% \mathrm{CO}_{2}$ in DMEM-STD in a humidified atmosphere for $24 \mathrm{~h}$ to facilitate attachment. The medium was then removed from each well and the cells were washed twice with $1 \mathrm{ml}$ of PBS (Life Technologies). The cells were then treated with $2.5 \mathrm{ml}$ per well of fresh low-Se medium. The cells were then cultured at $37^{\circ} \mathrm{C}$ with $5 \% \mathrm{CO}_{2}$ in a humidified atmosphere for $72 \mathrm{~h}$. The low-Se medium was then removed from each well and the cells were washed with $1 \mathrm{ml}$ of PBS. The cells were then treated with $2.5 \mathrm{ml}$ per well of fresh low-Se medium with or without $7 \mathrm{ng} / \mathrm{ml}$ ( $40 \mathrm{~nm}$ ) of sodium selenite (Sigma-Aldrich) for $16 \mathrm{~h}$ at $37^{\circ} \mathrm{C}$ with $5 \% \mathrm{CO}_{2}$ in a humidified atmosphere.

\section{Metabolic activity assay}

The effect of supplementation of $40 \mathrm{~nm}$ Se on the metabolic activity of CaCo-2 cells cultured in low Se for $72 \mathrm{~h}$ was measured using the water-soluble tetrazolium cytotoxicity assay (WST-1; Clontech) as described previously ${ }^{(53)}$. Metabolic activity was assessed at $T=0 \mathrm{~h}$ (low Se for $72 \mathrm{~h}$ ) and at $T=16 \mathrm{~h}$ (with or without $40 \mathrm{~nm}$ Se, as described above) on three biological replicates (with eight technical replicates each). Absorbance of the formazan dye produced was measured at 450 and $650 \mathrm{~nm}$ using a FlexStation 3 Spectrophotometer (Molecular Devices). For all measurements (including the blank), the background absorbance $(650 \mathrm{~nm})$ was subtracted from the detection wavelength $(450 \mathrm{~nm})$ and these corrected values were used for analysis.

\section{Real-time PCR assay}

The expression of the Se-sensitive genes, GPX1 and SEPW1, was quantified using hydrolysis-probe-based real-time PCR. After $16 \mathrm{~h}$ of low Se or treatment with $40 \mathrm{~nm}$ of sodium selenite, RNA was extracted using the SV Total RNA Isolation System (Promega) according to the manufacturer's instructions, with the quality and quantity of total RNA determined on the basis of 260:230 and 260:280 absorbance ratios using a NanoDrop 1000 Spectrophotometer (Thermo Fisher Scientific). A sample value of 1.8-2.0 for each ratio was considered to be of sufficient quality. The High-Capacity RNA-to-cDNA kit (Life Technologies) was used to prepare $2 \mu \mathrm{g}$ of complementary DNA (cDNA) from each RNA sample according to the manufacturer's instructions, and the cDNA samples were stored at $-20^{\circ} \mathrm{C}$ until required.

For real-time PCR analysis, the PrimeTime (Integrated DNA Technologies) assays were used. All PCR (no-template controls and samples) were prepared as quadruplicate $10 \mu \mathrm{l}$ reactions comprising a $9 \cdot 0-\mu \mathrm{l}$ aliquot of master mix $(5 \cdot 0 \mu \mathrm{l}$ of $2 \times \mathrm{Kapa}$ Probe Fast Mix (KK4703; Kapa Biosystems), $0.5 \mu$ l of $20 \times$ Integrated DNA Technologies (IDT) gene assay, $3.5 \mu$ of nucleasefree water (Life Technologies) and $1 \mu \mathrm{l}$ of cDNA (at a one in ten dilution in nuclease-free water). The thermal profile used was as follows: $95^{\circ} \mathrm{C}$ for $20 \mathrm{~s}$ followed by forty cycles of $95^{\circ} \mathrm{C}$ for $3 \mathrm{~s}$ and $60^{\circ} \mathrm{C}$ for $30 \mathrm{~s}$. The experiment was completed using a Rotor-Gene 6000 quantitative PCR instrument (Qiagen). These data were normalised to the geometric mean of the $\beta$-2 microglobulin (B2M) and the ribosomal protein L19 (RPL19) reference genes, with the application of the comparative quantification method with efficiency correction, and expressed as fold changes (stressed $v$. unstressed). The efficiencies for all PCR ranged between 1.93 and 2.01, where 2.0 represents $100 \%$ efficiency. qPCR data were ranked and analysed using the Kruskal-Wallis ANOVA method in SigmaStat 12.3 (Systat Software Inc.). Following ANOVA, significantly different means were identified using Tukey's post hoc test. All data with $P<0.05$ were considered to be statistically significant.

\section{Profiling of microRNA expression changes}

The NanoString nCounter technology ${ }^{(54)}$ was used to determine the absolute counts of 800 human miRNA (Human v2 miRNA Expression Assay; NanoString Technologies) in 150 ng of RNA from each sample, as described in the real-time qPCR method. The NanoString profiling was completed according to the manufacturer's instructions and the raw NanoString profiling data were normalised to the geometric mean of the top 100 expressing miRNA and the absolute number of counts for each miRNA was calculated using nSolver version 2.5 analysis 
software (NanoString Technologies). All data were analysed for statistical significance using Student's $t$ test in nSolver version 2.5 software. Data having $P<0.05$ were considered to be statistically significant.

\section{In silico data analyses}

To explore the effect(s) of Se-sensitive miRNA on biological pathways, we focused solely on miRNA-mRNA interactions, which were experimentally validated using the DIANA-TarBase version $7.0^{(55)}$. These data were then used to assess which pathways they were involved with on the basis of gene ontologies (Gene Ontology database ${ }^{(56-59)}$ ) and reaction events (Reactome database version $57^{(60,61)}$ ). These data were mapped using Ingenuity ${ }^{\circledR}$ Pathway Analysis software (IPA ${ }^{\circledR}$; Qiagen).

\section{Results}

\section{Culturing CaCo-2 cells in low selenium affects cell} metabolism and the expression of selenium status markers

To assess the effect of low Se on CaCo-2 viability and to confirm a low-Se environment, we measured metabolic activity (WST-1 assay) and the expression of GPX1 and SEPW1 genes ${ }^{(18,62)}$ (real-time qPCR). The mean metabolic activity of CaCo- 2 cells in a low-Se environment was measured to be 79 (SEM 6) \% of that of CaCo-2 in a 40-nm Se-supplemented environment. The qPCR data show that expression of the GPX1 and SEPW1 genes was significantly lower in a low-Se environment than in the 40-nм Se-supplemented one (-2.93-fold SEm 0.84, $P=0.010$ and $-2 \cdot 73$-fold sem $1.02, P=0.013$, respectively). As these genes have previously been shown to be highly sensitive to Se supply, these data show that the cells grown in the low-Se medium did have a low-Se status ${ }^{(18)}$; thus, other Se-sensitive genes may also have responded appropriately to the amount of Se present.

\section{Low selenium affects microRNA expression in CaCo-2 cells}

Of the 800 miRNA probes in the NanoString Codeset, the expression of ten miRNA was found to be affected by low Se (Table 1). On the basis of experimentally verified miRNA-mRNA interactions from the DIANA-TarBase version 7.0 database ${ }^{(55)}$, these ten Se-sensitive miRNA have 5364 interactions with 3588 distinct mRNA.

\section{Selenium-sensitive microRNA expression affects genes associated with cellular metabolism and signalling}

To explore the potential biological implications of the findings from Table 1, we utilised a range of open source databases for our analyses. First, we used the Gene Ontology database ${ }^{(56)}$ to identify the biological processes that the mRNA targeted by Se-sensitive miRNA are involved in, based on Protein ANnotation THrough Evolutionary Relationship (PANTHER) overrepresentation analysis ${ }^{(59)}$ (Fig. 1).

These data show that mRNA influenced by Se-sensitive miRNA are involved in key biological processes. While exploring the effects of Se-sensitive miRNA on mRNA on the basis of gene ontology pathways, the findings do not necessarily translate into biological interactions at the protein or the metabolite level. To clarify these effects, we further examined our data using the Reactome database, which uses reactions between entities (e.g. nucleic acids, proteins, complexes and small molecules) as the central point, rather than just gene interactions and associations.

\section{Selenium-sensitive microRNA affect genes associated with the cell cycle and the cellular response to stress}

On the basis of Reactome database analyses of the mRNA targeted by the ten Se-sensitive miRNA (Table 1), the cell cycle and the cellular response to stress pathways are, as expected, the most significant pathways affected as a consequence of the dysregulation of Se-sensitive miRNA (Table 2).

The genes within the cell cycle and the cellular response to stress pathways, which are experimentally validated targets of the ten Se-sensitive miRNA, are listed in Table 3. These data show that, of the ten Se-sensitive miRNA, mir-93-5p targets the majority of mRNA in these pathways, and that several histonecluster genes (particularly the histone $\mathrm{H} 4$ cluster) are the largest family of mRNA that are targeted.

Table 1. The ten microRNA (miRNA) whose expression is altered by a low-selenium environment in the CaCo-2 cell line* (Mean expression levels (EL) with their standard errors)

\begin{tabular}{|c|c|c|c|c|c|}
\hline \multirow[b]{2}{*}{ Mature miRNA } & \multirow[b]{2}{*}{ Accession ID } & \multicolumn{4}{|c|}{ Low Se $v$. adequate Se } \\
\hline & & Mean EL & SEM & $P$ & miRNA-mRNA \\
\hline hsa-miR-93-5p & MIMAT0000093 & 0.48 & 0.14 & 0.037 & 2071 \\
\hline hsa-miR-106a-5p & MIMAT0000103 & 0.24 & 0.02 & 0.005 & 1196 \\
\hline hsa-miR-205-5p & MIMAT0000266 & 0.68 & 0.15 & 0.024 & 368 \\
\hline hsa-miR-200c-3p & MIMAT0000617 & 0.74 & 0.19 & 0.036 & 835 \\
\hline hsa-miR-99b-5p & MIMAT0000689 & 0.50 & 0.14 & 0.029 & 183 \\
\hline hsa-miR-302d-3p & MIMAT0000718 & 1.81 & 0.09 & 0.013 & 231 \\
\hline hsa-miR-373-3p & MIMAT0000726 & 0.66 & 0.08 & 0.006 & 134 \\
\hline hsa-miR-483-3p & MIMAT0002173 & 0.74 & 0.18 & 0.041 & 199 \\
\hline hsa-miR-512-5p & MIMAT0002822 & 0.72 & 0.17 & 0.035 & 59 \\
\hline hsa-miR-4454 & MIMAT0018976 & 0.41 & 0.14 & 0.039 & 88 \\
\hline
\end{tabular}

miRNA-mRNA, the number of unique interactions for a given miRNA within the overall 5364 experimentally supported interactions.

* EL compared with adequate Se $(40 \mathrm{~nm}, \mathrm{EL}=1.0)$, of three independent replicates and was generated using nSolver (version 2.5$)$ software and the DIANA-TarBase version 7.0 database ${ }^{(55)}$. 
Selenium-sensitive microRNA

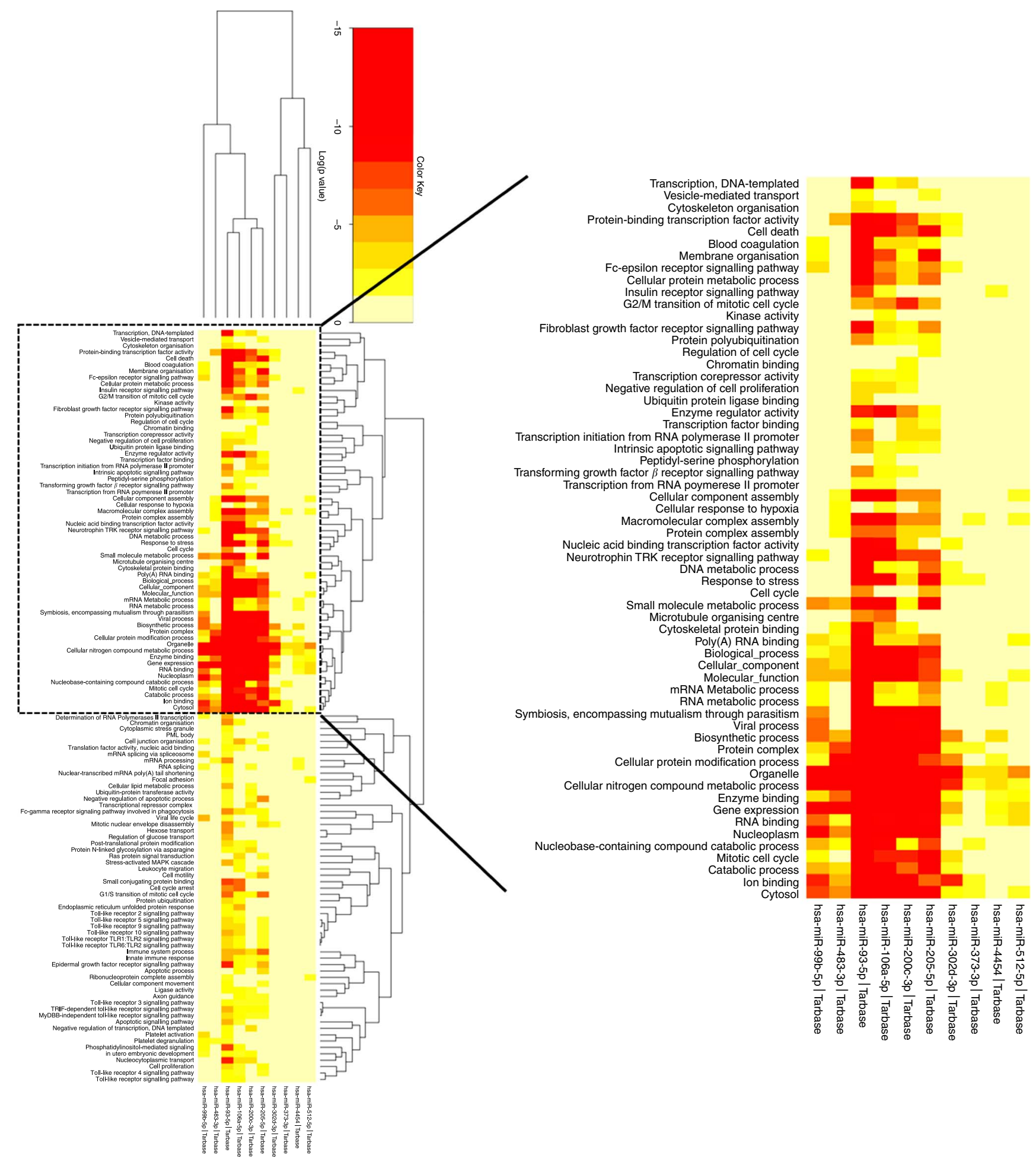

Fig. 1. A heat map showing the gene ontologies affected by expression of selenium-responsive microRNA (miRNA). The intensity of the colours represents the log ( $P$ value) of the probability that the association of miRNA-mRNA in a particular pathway occurred by chance. The lower the log $(P$ value) the less likely these associations occur by chance (indicated in red).

These data show that considering both gene-based pathway mapping (e.g. gene ontologies) and reaction events (e.g. Reactome data) provides a more comprehensive analyses of the consequences of genes targeted by Se-sensitive miRNA.
Selenium-sensitive microRNA dysregulation may affect appropriate regulation of cancer pathways

To explore the effect of dysregulated Se-sensitive miRNA (Table 1) on genes associated with cancer pathways, 
Table 2. A summary of the significant high-level reactome pathways containing genes whose expression is influenced by the selenium-sensitive microRNA shown in Table $1^{*}$

\begin{tabular}{llccc}
\hline Pathway ID & Pathway name & Entities $\dagger$ & $P$ & Reactionsł \\
\hline R-HSA-1640170 & Cell cycle & 237 & 0.018 & 91.49 \\
R-HSA-2262752 & Cellular responses to stress & 177 & 0.024 & 84.39 \\
\hline
\end{tabular}

* Data were derived from the Reactome database ${ }^{(60)}$.

$\dagger$ The number of mapped entities, in this case genes that match the pathway.

$\ddagger$ The percentage of reactions in a specified pathway with at least one mapped entity in the data set.

Table 3. The selenium-sensitive microRNA (miRNA) whose expression is altered by a low-selenium environment and their mRNA targets in the Reactome pathways (Table 2)

\begin{tabular}{|c|c|c|}
\hline Reactome pathways & Low-Se miRNA & Associated mRNA in Reactome pathway \\
\hline \multirow[t]{8}{*}{ Cell cycle (R-HSA-1640170) } & miR-106a-5p $(F C=-4 \cdot 17)$ & $\begin{array}{l}\text { CCNB1, CDKN1A, HERC2, MDM4, PSMD13, PSME3, PSMF1, UBC, WEE1, WHSC1, YWHAB, } \\
\text { ZNF385A }\end{array}$ \\
\hline & $\begin{array}{l}\operatorname{miR}-200 c-3 p(F C=-1.59) \\
\operatorname{miR}-205-5 p(F C=-1.66)\end{array}$ & $\begin{array}{l}\text { BRCA1, CCNE2, CDK2, CDKN1B, MCM8, MDM4, PSMB4, RNF168, WEE1, WHSC1, YWHAG } \\
\text { CCNB1, CCNB2, CDC20, CDC23, CDK1, PSMB8, PSMD1, PSMD13, PSMD2, PSMD7, PSME2, } \\
\text { RNF168, RPA2, TP53, TP53BP1 }\end{array}$ \\
\hline & miR-302d-3p $(F C=1.81)$ & CCNB1, CDK2, PSMB2, RAD1, ZNF385A \\
\hline & miR-373-3p $(F C=-1.57)$ & MRE11A \\
\hline & $\mathrm{miR}-4454(\mathrm{FC}=-3.03)$ & YWHAB \\
\hline & $\operatorname{miR}-483-3 p(F C=-1.63)$ & ANAPC11, MDM4, UBE2C \\
\hline & $\operatorname{miR}-93-5 p(F C=-2 \cdot 43)$ & $\begin{array}{l}\text { ANAPC11, ANAPC5, BRE, CCNB1, CDC16, CDC6, CDKN1A, CDKN1B, FAM175A, H2AFX, } \\
\text { HIST1H2BD, HIST1H4A, HIST1H4B, HIST1H4C, HIST1H4D, HIST1H4E, HIST1H4H, HIST1H4I, } \\
\text { HIST1H4J, HIST1H4K, HIST1H4L, HIST2H4A, HIST2H44, HIST4H4, HUS1, MAD2L1, MCM3, } \\
\text { MCM5, MDM2, MDM4, MRE11A, PIAS4, PKMYT1, PSMA4, PSMA7, PSMB4, PSMC1, PSMD11, } \\
\text { PSMD4, RBBP8, RFC3, RPA2, RPS27A, TP53, UBC, UBE2E1, WEE1, WHSC1, YWHAB, } \\
\text { YWHAQ, YWHAZ, ZNF385A }\end{array}$ \\
\hline & $\operatorname{miR}-99 b-5 p(F C=-2 \cdot 32)$ & CHEK1, HIST1H2BK, MCM3, MCM4 \\
\hline \multirow[t]{8}{*}{$\begin{array}{l}\text { Cellular responses to stress } \\
\text { (R-HSA-2262752) }\end{array}$} & miR-106a-5p $(F C=-4 \cdot 17)$ & $\begin{array}{l}\text { AGO1, ARNT, ASF1A, ATG16L1, BAG5, CDKN1A, CDKN2C, CEBPB, CHMP3, CXCL8, E2F1, } \\
\text { EGLN1, EGLN3, EPAS1, ERO1A, ETS1, HIST1H3B, HMGA1, HMGA2, IL1A, LAMTOR1, MAPK1, } \\
\text { MAPK7, MAPK8, MAPKAPK3, MINK1, MTMR3, NUP205, NUP58, PHC2, PHC3, PIK3C3, PRDX3, } \\
\text { PRKAB2, RANBP2, RB1, RBBP7, RPS6KA2, RPS6KA3, RRAGD, SEH1L, SIRT1, SOD2, STAT3, } \\
\text { TNRC6A, TNRC6B, TXN, UBC, UBE2D3, ULK1, VEGFA }\end{array}$ \\
\hline & miR-200c-3p $(F C=-1.59)$ & $\begin{array}{l}\text { ATG12, BAG5, BMI1, CBX4, CCNE2, CDK2, CDKN1B, CEBPB, CHMP2B, E2F3, EP300, ETS1, } \\
\text { H2AFV, HSPA13, HSPA8, JUN, LMNB1, NUP160, NUP58, PHC3, RANBP2, RNF2, RPS6KA3, } \\
\text { SIRT1, SUZ12, TERF2, TSC1, VEGFA }\end{array}$ \\
\hline & $\operatorname{miR}-205-5 p(F C=-1.66)$ & $\begin{array}{l}\text { CDC23, CHMP2A, DNAJC7, E2F1, EED, GABARAPL1, HSPA8, HSPA9, NFKB1, NUP37, RBX1, } \\
\text { RPA2, RPS19BP1, RRAGA, SEH1L, TERF2IP, TP53, UBE2D3, VEGFA, VHL }\end{array}$ \\
\hline & $\begin{array}{l}\operatorname{miR}-302 d-3 p(F C=1.81) \\
\operatorname{miR}-373-3 p(F C=-1.57)\end{array}$ & $\begin{array}{l}\text { AGO3, BECN1, CDK2, MINK1, NUP88, RANBP2, SUZ12, TNRC6B, VEGFA } \\
\text { CXCL8, HIF1A, HIF1AN, HSPA14, LMNB1, MRE11A, NUP153, NUP58, PHC2, PHC3, PRKAA1, } \\
\text { RELA, TNRC6A, VEGFA }\end{array}$ \\
\hline & $\begin{array}{l}\operatorname{miR}-4454(F C=-3.03) \\
\operatorname{miR}-483-3 p(F C=-1.63)\end{array}$ & $\begin{array}{l}\text { CCAR2, HSPA1A, LAMTOR1, POT1, PRKAA1 } \\
\text { ANAPC11, EGLN2, EZH2, GSK3B, HMGA1, MAP1LC3B, MAPKAPK2, PHC3, PRKAA1, UBE2C, } \\
\text { WDR45B }\end{array}$ \\
\hline & miR-512-5p $(F C=-1.63)$ & DNAJB1 \\
\hline & $\operatorname{miR}-93-5 p(F C=-2.43)$ & $\begin{array}{l}\text { ACD, AGO1, AGO3, AGO4, AMBRA1, ANAPC11, ANAPC5, ASF1A, ATG16L1, ATG3, BAG2, CBX2, } \\
\text { CBX6, CBX8, CDC16, CDK4, CDKN1A, CDKN1B, CDKN2C, CHMP3, CITED2, CXCL8, E2F1, } \\
\text { EEF1A1, EGLN3, EP400, ERO1A, EZH2, GSK3B, H1F0, H2AFV, H2AFX, HIF1A, HIST1H2BD, } \\
\text { HIST1H3A, HIST1H3B, HIST1H3C, HIST1H3D, HIST1H3E, HIST1H3I, HIST1H3J, HIST1H4A, } \\
\text { HIST1H4B, HIST1H4C, HIST1H4D, HIST1H41 HIST HIST1H4H, HIST1H4I, HIST1H4J, HIST1H4K, } \\
\text { HIST1H4L, HIST2H3A, HIST2H3C, HIST2H3D, HIST2H4A, HIST2H4B, HIST4H4, HMGA2, } \\
\text { HSBP1, HSP90AA1, HSPA4L, HSPA8, JUN, LAMTOR1, LAMTOR5, LMNB1, MAP3K5, MAPK1, } \\
\text { MAPK7, MAPK8, MAPK9, MDM2, MINK1, MRE11A, MTMR3, NUP107, NUP153, NUP188, } \\
\text { NUP205, NUP58, NUP62, NUPL2, PHC2, PHC3, PIK3C3, PRDX3, PRDX6, PRKAA2, PRKAB2, } \\
\text { PTGES3, RAE1, RANBP2, RB1, RBBP7, RNF2, RPA2, RPS27A, RPS6KA3, RRAGD, SOD2, SP1, } \\
\text { STAT3, SUZ12, TNRC6A, TNRC6B, TNRC6C, TP53, TPR, TSC1, TXN, UBC, UBE2D3, UBE2E1, } \\
\text { UBN1, ULK1, VCP, VEGFA, VHL, WIPI2 }\end{array}$ \\
\hline & miR-99b-5p $(F C=-2 \cdot 32)$ & $\begin{array}{l}\text { CBX6, CEBPB, ERF, H2AFZ, HIST1H1C, HIST1H2BK, MTOR, NUP43, SP1, TNRC6B, UBE2D2, } \\
\text { VEGFA }\end{array}$ \\
\hline
\end{tabular}

FC, fold change.

we mapped the mRNA targets of these miRNA onto the Wnt/ $\beta$-catenin, p53 and ERK/MAPK pathways using Ingenuity Pathway Analysis (Fig. 2-4). These data show that several genes, for example, AKT, ERK1/2, MDM2, PIK3, RAS and p53, in key colon cancer-associated pathways are targeted by Se-sensitive miRNA (Table 1), indicating that fluctuations in Se status may affect signalling pathways linked to colon cancer development and progression.

\section{Discussion}

In our study, we hypothesised that there are miRNA whose expression is dysregulated in a low-Se environment ( $<40 \mathrm{~nm}$ ), and that this dysregulation explains in part the effects of Se status on key biological pathways. We have shown that there are ten miRNA, which have been experimentally validated in the literature, to target mRNA involved in key metabolic and 


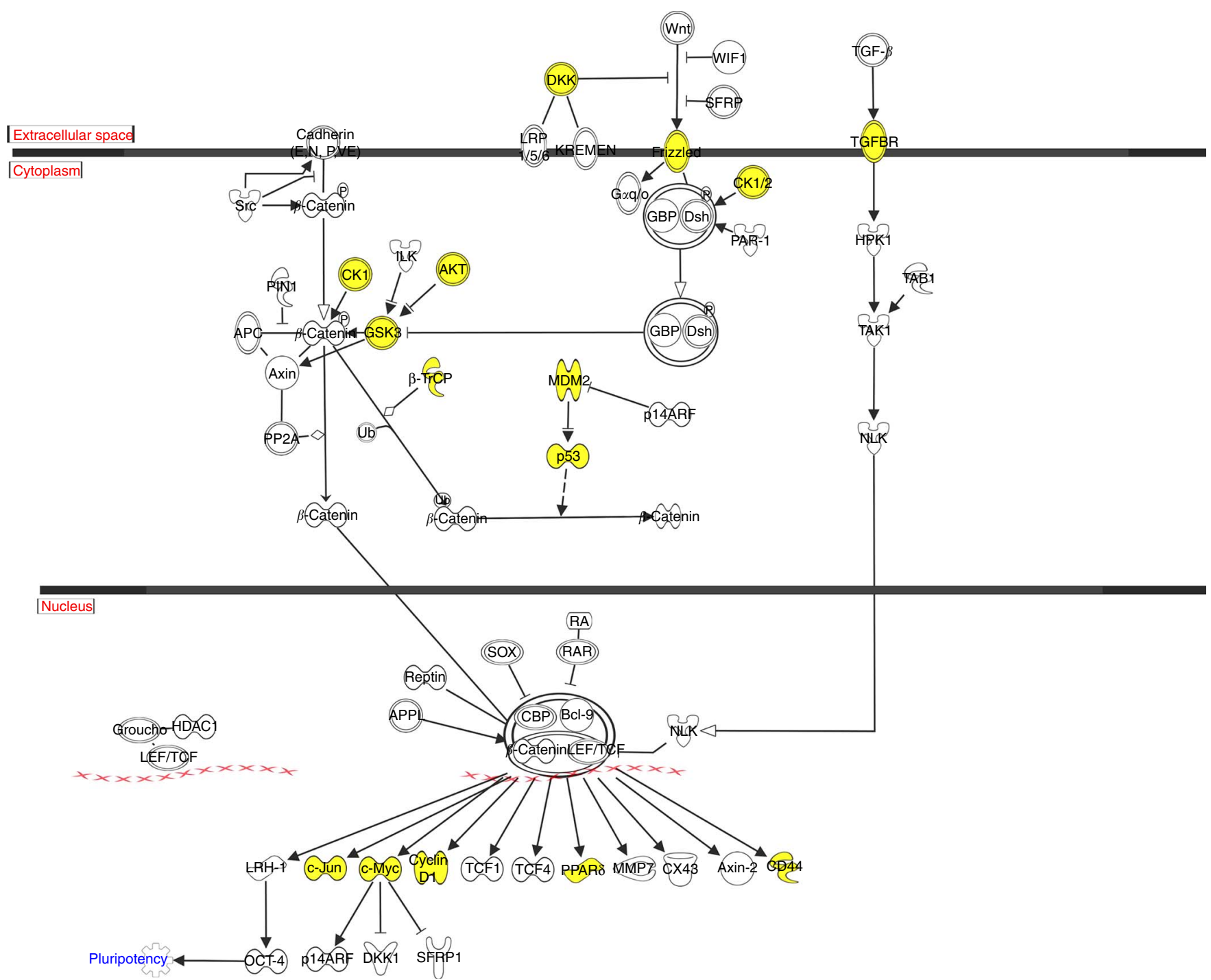

Fig. 2. Effect of dysregulated selenium-sensitive miRNA (Table 1) on genes associated with the Wnt/ $\beta$-catenin pathway. Genes targeted by selenium-sensitive miRNA are highlighted in yellow. These data were generated through the use of IPA (Ingenuity $®$ Systems, www.ingenuity.com).

signalling pathways (Table 3) and that canonical signalling pathways in colon cancer are sensitive to Se (Fig. 2-4). To our knowledge, these data are the first reported on Se-sensitive miRNA on the basis of direct miRNA counts rather than on the basis of relative quantification-based technologies such as microarray and real-time qPCR. Furthermore, we explored the pathways/processes that these miRNA targeted, considering only their experimentally validated mRNA targets, on the basis of gene ontologies and Reactome data. Importantly, our data show that the effects of low Se on biological pathways may, in part, be due to the dysregulation of the ten Se-sensitive miRNA identified.

A previous study on the effects of Se status on miRNA used microarray and real-time qPCR methods ${ }^{(32)}$ and found that thirty-four miRNA and fifty mRNA were dysregulated in low-Se conditions in vitro. In contrast to our data, none of the Se-sensitive miRNA reported in Table 1 was found to be affected in that study. However, twenty-eight of these previously observed
miRNA were found in our data set, and despite not being differently expressed in our data, the magnitude of the response in expression was consistent. Interestingly, seven of the mRNA (ATP1B3, CACYBP, CMIP, DCUN1D4, GPX4, MT1F and SEPHS2) that showed altered expression are also targets of Se-sensitive miRNA in our data set, despite little overlap in miRNA data ${ }^{(32)}$. Discrepancies between the two studies might be explained by the use of different detection technologies (microarray $v$. NanoString) and the updated Genome Reference Consortium Human build 38 used in this study.

The data from bioinformatic analysis (Table 3) show that key genes in the cell cycle and the cellular response to stress are regulated by the Se-sensitive miRNA identified in this study. With respect to the cell cycle, genes regulating cell cycle progression, for example, cyclins (B1, B2, E2), cyclin-dependent kinases (1 and 2) and cyclin-dependent kinase inhibitors (1A), checkpoints (E2F1 and RB), and DNA licencing for replication (MCM2 and MCM5) are influenced by Se-sensitive miRNA. 


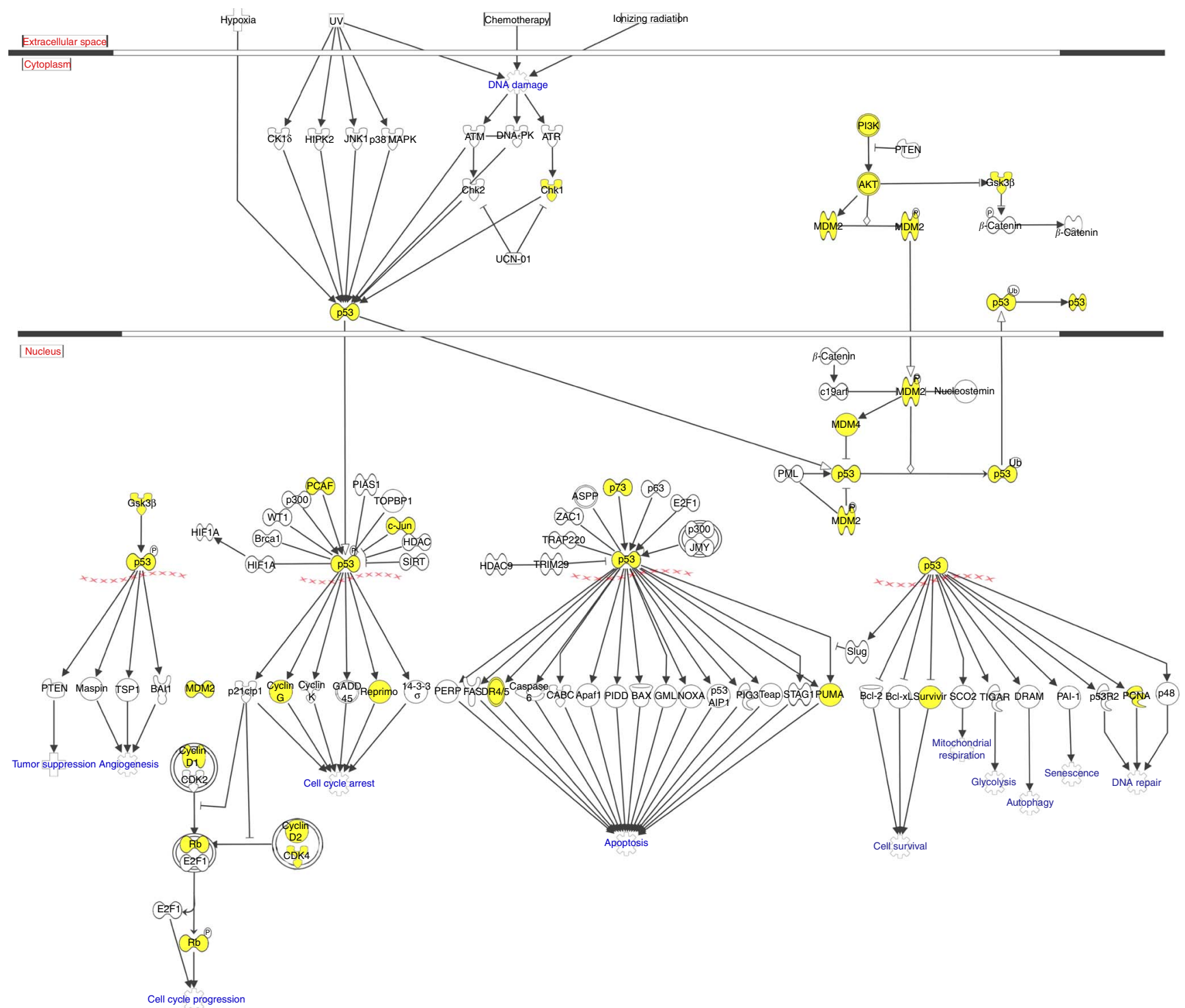

Fig. 3. Effect of dysregulated selenium-sensitive miRNA (Table 1) on genes associated with the p53 pathway. Genes targeted by selenium-sensitive miRNA are highlighted in yellow. These data were generated through the use of IPA (Ingenuity $\circledast$ Systems, www.ingenuity.com).

Previous studies ${ }^{(12,15,16)}$ have reported that Se status influences the expression of these genes in disease, and the appropriate regulation of the cell cycle by both mRNA and miRNA is essential for preventing carcinogenic transformation. The bioinformatic analysis also found that Se-sensitive miRNA regulate the expression of genes involved in the cellular response to stress, for example, antioxidant enzymes (SOD2), mitogen-activated signalling cascades (MAPK1, MAPK7 and MAPK8) and in the heat-shock stress responses (HSPA8, HSPA9 and HSPA13). Se status is known to influence several stress responses, such as those caused by oxidative and unfolded protein stress ${ }^{(63-65)}$, and our data suggest that Se-sensitive miRNA may be influential in Se-mediated stress responses.

We have also found that in $\mathrm{CaCo}-2$ cells, inadequate Se supply alters the expression profile of miRNA that regulate the expression of key genes in the canonical Wnt $/ \beta$-catenin, $\mathrm{p} 53$ and ERK/MAPK pathways (Fig. 2-4). Previous studies have shown a link between Se status and the expression of genes in these pathways ${ }^{(12-16,42,44-48)}$; however, they did not assess the effect of altered miRNA expression in response to Se status on the genes that were Se sensitive in these pathways. Our data suggest that fluctuations in Se status due to diet or ill health ${ }^{(5,7)}$, which result in dysregulated miRNA expression, may affect the appropriate functioning of these pathways, influencing pathogenesis in the colon.

Inadequate Se levels and the potential cellular dysfunction associated with them may increase the risk for disease. Se supply can be affected by compromised intestinal function - for example, by inflammatory bowel disease ${ }^{(66)}$, or by altered activity of key genes due to $\mathrm{SNP}^{(5)}$ - such that the consequences of fluctuating Se status, exacerbated by inappropriate Se-sensitive miRNA-mediated regulation, may have an important role in how intestinal diseases such as cancer develop and progress. 


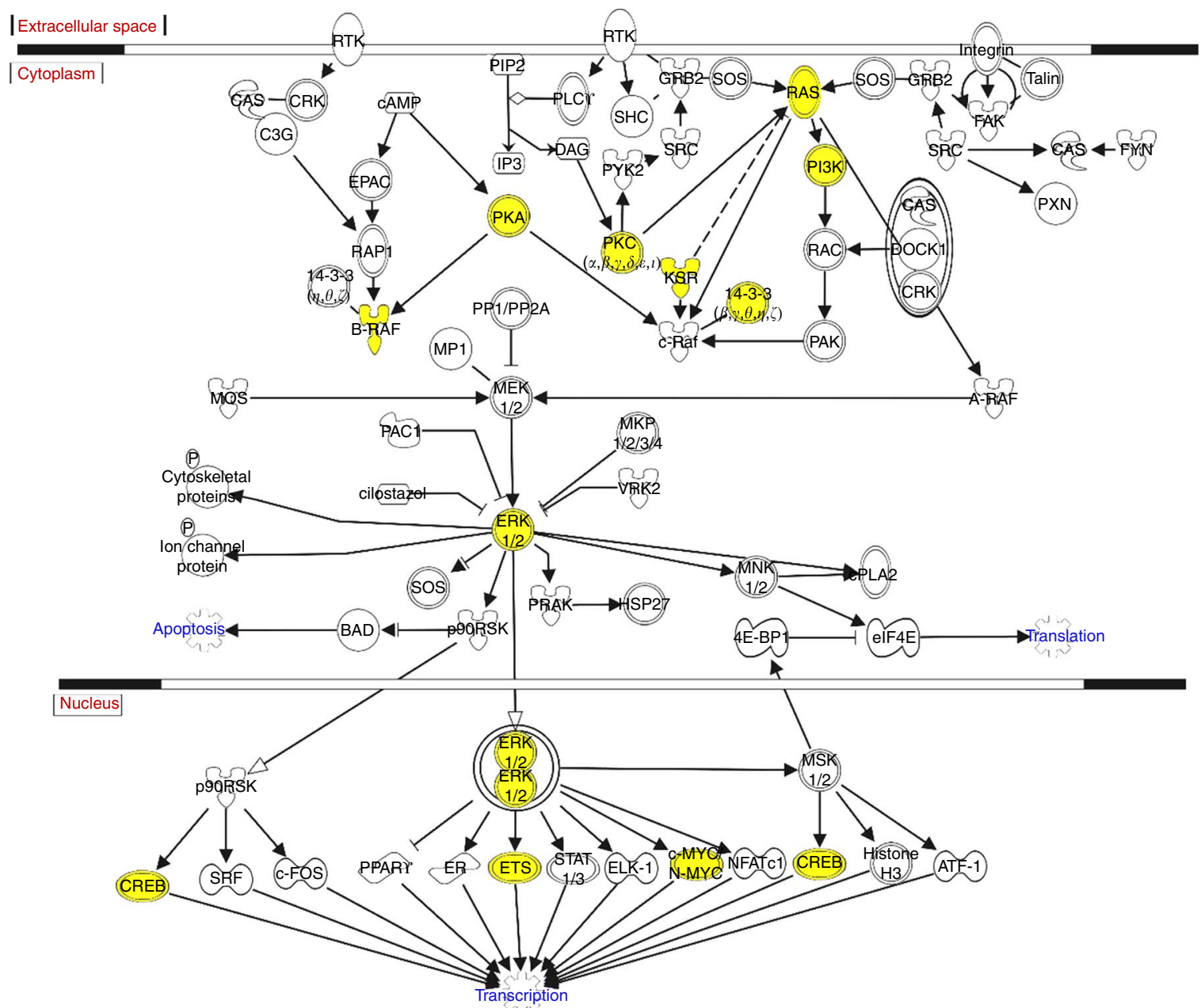

Fig. 4. Effect of dysregulated selenium-sensitive miRNA (Table 1) on genes associated with the ERK/MAPK pathway. Genes targeted by selenium-sensitive miRNA are highlighted in yellow. These data were generated through the use of IPA (Ingenuity® Systems, www.ingenuity.com).

Although our study has identified that ten miRNA are sensitive to Se status and that these miRNA target mRNA involved in the cell cycle, cell stress response and in key cancer-associated pathways, these findings require further validation. Our data are based on experimental profiling of miRNA abundance and in silico mRNA target analysis (based on experimentally validated miRNA-mRNA interactions, but not necessarily in the cell line used for this study). To robustly demonstrate that these Se miRNA directly influence the mRNA and biological pathways identified in this study, further functional validation, such as protein abundance/activity analysis, is required. However, our data provide a foundation on which to further study the influence of Se status on miRNA-mediated regulation of biological pathways.

\section{Conclusions}

Using a novel direct quantification method, we have identified ten miRNA that are Se sensitive, which have been experimentally validated in the literature to target mRNA involved in key metabolic, signalling and canonical colon cancer pathways. Importantly, our data represent important preliminary knowledge of how Se status affects miRNA expression and how these changes may affect biological pathways. First, the data show that the effects of low Se on biological pathways may, in part, be due to the dysregulation of the ten Se-sensitive miRNA identified. Second, the data identify the cell cycle and the stress response pathways as being potentially affected by Se status through miRNA expression. These findings contribute to an understanding of how the regulation of miRNA by Se supply could potentially influence the cells' response to further challenges that may ultimately lead to disease.

\section{Acknowledgements}

This study was co-funded by the MICROGENNET exchange programme funded by the EU Marie Curie International Research Staff Exchange Scheme grant and the Royal Society of New Zealand. Additional funding was provided by AgResearch Ltd (Core funding - Food Nutrition Core programme) and by the New Zealand Ministry of Business, Innovation and Employment (Nutrigenomics New Zealand programme). The funding sponsors had no role in the design of the study; in the collection, analyses or interpretation of 
data; in the writing of the manuscript; and in the decision to publish the results.

M. J. M. and J. E. H. devised the experiments; M. J. M. and K. R. completed the experimental work; M. J. M. completed the data analysis and wrote the manuscript, with direct input from J. E. H., K. R. and N. C. R.

The authors declare that there are no conflicts of interest.

\section{References}

1. Rayman MP (2012) Selenium and human health. Lancet 379, $1256-1268$.

2. Roman M, Jitaru P \& Barbante C (2014) Selenium biochemistry and its role for human health. Metallomics 6, 25-54.

3. Lobanov AV, Hatfield DL \& Gladyshev VN (2009) Eukaryotic selenoproteins and selenoproteomes. Biochim Biophys Acta 1790, 1424-1428.

4. Hesketh J \& Méplan C (2011) Transcriptomics and functional genetic polymorphisms as biomarkers of micronutrient function: focus on selenium as an exemplar. Proc Nutr Soc $\mathbf{7 0}$, 365-373.

5. Méplan C \& Hesketh J (2012) The influence of selenium and selenoprotein gene variants on colorectal cancer risk. Mutagenesis 27, 177-186.

6. Méplan C \& Hesketh J (2014) Selenium and cancer: a story that should not be forgotten - insights from genomics. Cancer Treat Res 159, 145-166.

7. Méplan C (2015) Selenium and chronic diseases: a nutritional genomics perspective. Nutrients 7, 3621-3651.

8. Barrett CW, Reddy VK, Short SP, et al. (2015) Selenoprotein P influences colitis-induced tumorigenesis by mediating stemness and oxidative damage. J Clin Invest 125, 2646-2660.

9. Hazane-Puch F, Arnaud J, Trocmé C, et al. (2016) Sodium selenite decreased HDAC activity, cell proliferation and induced apoptosis in three human glioblastoma cells. Anticancer Agents Med Chem 16, 490-500.

10. Liu W, Yao H, Zhao W, et al. (2016) Selenoprotein W was correlated with the protective effect of selenium on chicken myocardial cells from oxidative damage. Biol Trace Elem Res 171, 419-426.

11. Li Z, Meng J, Xu TJ, et al. (2013) Sodium selenite induces apoptosis in colon cancer cells via Bax-dependent mitochondrial pathway. Eur Rev Med Pharmacol Sci 17, 2166-2171.

12. Hawkes WC, Printsev I \& Alkan Z (2012) Selenoprotein W depletion induces a p53- and p21-dependent delay in cell cycle progression in RWPE-1 prostate epithelial cells. $J$ Cell Biochem 113, 61-69.

13. Wu M, Wu RT, Wang TT, et al. (2011) Role for p53 in seleniuminduced senescence. J Agric Food Chem 59, 11882-11887.

14. Wu M, Kang MM, Schoene NW, et al. (2010) Selenium compounds activate early barriers of tumorigenesis. $\mathrm{J} \mathrm{Biol} \mathrm{Chem}$ 285, 12055-12062.

15. Wu RT, Cao L, Chen BP, et al. (2014) Selenoprotein H suppresses cellular senescence through genome maintenance and redox regulation. J Biol Chem 289, 34378-34388.

16. Hawkes WC \& Alkan Z (2011) Delayed cell cycle progression from SEPW1 depletion is p53- and p21-dependent in MCF-7 breast cancer cells. Biochem Biophys Res Commun 413, 36-40.

17. Tsuji PA, Naranjo-Suarez S, Carlson BA, et al. (2011) Deficiency in the $15 \mathrm{kDa}$ selenoprotein inhibits human colon cancer cell growth. Nutrients 3, 805-817.

18. Pagmantidis V, Bermano G, Villette S, et al. (2005) Effects of Se-depletion on glutathione peroxidase and selenoprotein W gene expression in the colon. FEBS Lett 579, 792-796.
19. Lipinski B (2017) Sodium selenite as an anti-cancer agent. Anticancer Agents Med Chem 17, 658-661.

20. Vinceti M, Dennert G, Crespi CM, et al. (2014) Selenium for preventing cancer. The Cochrane Database of Systematic Reviews, issue 3, CD005195.

21. Bartel DP (2009) MicroRNAs: target recognition and regulatory functions. Cell 136, 215-233.

22. Friedman RC, Farh KK, Burge CB, et al. (2009) Most mammalian mRNAs are conserved targets of microRNAs. Genome Res 19, 92-105.

23. Voorhoeve PM (2010) MicroRNAs: oncogenes, tumor suppressors or master regulators of cancer heterogeneity? Biochim Biophys Acta 1805, 72-86.

24. Zhang B, Pan X, Cobb GP, et al. (2007) MicroRNAs as oncogenes and tumor suppressors. Dev Biol 302, 1-12.

25. Hata A \& Lieberman J (2015) Dysregulation of microRNA biogenesis and gene silencing in cancer. Sci Signal $\mathbf{8}$, re3.

26. Shah MS, Kim E, Davidson LA, et al. (2016) Comparative effects of diet and carcinogen on microRNA expression in the stem cell niche of the mouse colonic crypt. Biochim Biophys Acta 1862, 121-134.

27. Ramalingam S, Subramaniam D \& Anant S (2015) Manipulating miRNA expression: a novel approach for colon cancer prevention and chemotherapy. Curr Pharmacol Rep 1, 141-153.

28. Ross SA \& Davis CD (2014) The emerging role of microRNAs and nutrition in modulating health and disease. Annu Rev Nutr 34, 305-336.

29. Karius T, Schnekenburger M, Dicato M, et al. (2012) MicroRNAs in cancer management and their modulation by dietary agents. Biochem Pharmacol 83, 1591-1601.

30. Shah MS, Davidson LA \& Chapkin RS (2012) Mechanistic insights into the role of microRNAs in cancer: influence of nutrient crosstalk. Front Genet 3, 305.

31. Shah MS, Schwartz SL, Zhao C, et al. (2011) Integrated microRNA and mRNA expression profiling in a rat colon carcinogenesis model: effect of a chemo-protective diet. Physiol Genomics 43, 640-654.

32. Maciel-Dominguez A, Swan D, Ford D, et al. (2013) Selenium alters miRNA profile in an intestinal cell line: evidence that miR-185 regulates expression of GPX2 and SEPSH2. Mol Nutr Food Res 57, 2195-2205.

33. Ferlay J, Soerjomataram I, Dikshit R, et al. (2015) Cancer incidence and mortality worldwide: sources, methods and major patterns in GLOBOCAN 2012. Int J Cancer 136, E359-E386.

34. Schneikert J \& Behrens J (2007) The canonical Wnt signalling pathway and its APC partner in colon cancer development. Gut 56, 417-425.

35. MacDonald BT, Tamai K \& He X (2009) Wnt/beta-catenin signaling: components, mechanisms, and diseases. Dev Cell 17, 9-26.

36. Kim EK \& Choi EJ (2010) Pathological roles of MAPK signaling pathways in human diseases. Biochim Biophys Acta 1802, 396-405.

37. Tachibana M, Kawamata H, Fujimori T, et al. (2004) Dysfunction of p53 pathway in human colorectal cancer: analysis of p53 gene mutation and the expression of the p53-associated factors p14ARF, p33ING1, p21WAF1 and MDM2. Int J Oncol 25, 913-920.

38. Colussi D, Brandi G, Bazzoli F, et al. (2013) Molecular pathways involved in colorectal cancer: implications for disease behavior and prevention. Int J Mol Sci 14, 16365-16385.

39. Jiricny J \& Marra G (2003) DNA repair defects in colon cancer. Curr Opin Genet Dev 13, 61-69.

40. Schetter AJ \& Harris CC (2011) Alterations of microRNAs contribute to colon carcinogenesis. Semin Oncol 38, 734-742. 
41. Hutchison J, Cohen Z, Onyeagucha BC, et al. (2013) How microRNAs influence both hereditary and inflammatorymediated colon cancers. Cancer Genet 206, 309-316.

42. Bera S, De Rosa V, Rachidi W, et al. (2013) Does a role for selenium in DNA damage repair explain apparent controversies in its use in chemoprevention? Mutagenesis 28, $127-134$

43. Lewis BP, Shih IH, Jones-Rhoades MW, et al. (2003) Prediction of mammalian microRNA targets. Cell 115, 787-798.

44. Barrett CW, Reddy VK, Short SP, et al. (2015) Selenoprotein P influences colitis-induced tumorigenesis by mediating stemness and oxidative damage. J Clin Invest 125, 2646-2660.

45. Tsuji PA, Carlson BA, Yoo MH, et al. (2015) The $15 \mathrm{kDa}$ selenoprotein and thioredoxin reductase 1 promote colon cancer by different pathways. PLOS ONE 10, e0124487.

46. Brigelius-Flohé R \& Kipp AP (2013) Selenium in the redox regulation of the Nrf2 and the Wnt pathway. Methods Enzymol $\mathbf{5 2 7}, 65-86$

47. Kipp AP, Müller MF, Göken EM, et al. (2012) The selenoproteins GPx2, TrxR2 and TrxR3 are regulated by Wnt signalling in the intestinal epithelium. Biochim Biophys Acta 1820, 1588-1596.

48. Xing Y, Liu Z, Yang G, et al. (2015) MicroRNA expression profiles in rats with selenium deficiency and the possible role of the $\mathrm{Wnt} / \beta$-catenin signaling pathway in cardiac dysfunction. Int J Mol Med 35, 143-152.

49. Luan Y, Zhao J, Yao H, et al. (2016) Selenium deficiency influences the mRNA expression of selenoproteins and cytokines in chicken erythrocytes. Biol Trace Elem Res $\mathbf{1 7 1}$, 427-436.

50. Schomburg L \& Schweizer U (2009) Hierarchical regulation of selenoprotein expression and sex-specific effects of selenium. Biochim Biophys Acta 1790, 1453-1462.

51. Berry MJ (2005) Insights into the hierarchy of selenium incorporation. Nat Genet 37, 1162-1163.

52. Bermano G, Arthur JR \& Hesketh JE (1996) Selective control of cytosolic glutathione peroxidase and phospholipid hydroperoxide glutathione peroxidase mRNA stability by selenium supply. FEBS Lett 387, 157-160.

53. McCann MJ, Rowland IR \& Roy NC (2014) The anti-proliferative effects of enterolactone in prostate cancer cells: evidence for the role of DNA licencing genes, miR-106b cluster expression, and PTEN dosage. Nutrients 6, 4839-4855.

54. Geiss GK, Bumgarner RE, Birditt B, et al. (2008) Direct multiplexed measurement of gene expression with colorcoded probe pairs. Nat Biotechnol 26, 317-325.

55. Vlachos IS, Paraskevopoulou MD, Karagkouni D, et al. (2015) DIANA-TarBase v7.0: indexing more than half a million experimentally supported miRNA:mRNA interactions. Nucleic Acids Res 43, D153-D159.

56. Gene Ontology Consortium (2017) Gene Ontology Consortium database. http://geneontology.org (accessed April 2017).

57. Ashburner M, Ball CA, Blake JA, et al. (2000) Gene ontology: tool for the unification of biology. Nat Genet 25, 25-29.

58. Gene Ontology Consortium (2013) Gene Ontology annotations and resources. Nucleic Acids Res 41, D530-D535.

59. Mi H, Poudel S, Muruganujan A, et al. (2016) PANTHER version 10: expanded protein families and functions, and analysis tools. Nucleic Acids Res 44, D336-D342.

60. Reactome (2017) Reactome Pathway Database. http://www. reactome.org (accessed April 2017).

61. Croft D, O'Kelly G, Wu G, et al. (2011) Reactome: a database of reactions, pathways and biological processes. Nucleic Acids Res 39, D691-D697.

62. Kipp A, Banning A, van Schothorst EM, et al. (2009) Four selenoproteins, protein biosynthesis, and Wnt signalling are particularly sensitive to limited selenium intake in mouse colon. Mol Nutr Food Res 53, 1561-1572.

63. Wu Y, Zhang H, Dong Y, et al. (2005) Endoplasmic reticulum stress signal mediators are targets of selenium action. Cancer Res 65, 9073-9079.

64. de Rosa V, Erkekoğlu P, Forestier A, et al. (2012) Low doses of selenium specifically stimulate the repair of oxidative DNA damage in LNCaP prostate cancer cells. Free Radic Res $\mathbf{4 6}$, 105-116.

65. Touat-Hamici Z, Legrain Y, Bulteau AL, et al. (2014) Selective up-regulation of human selenoproteins in response to oxidative stress. J Biol Chem 289, 14750-14761.

66. Kudva AK, Shay AE \& Prabhu KS (2015) Selenium and inflammatory bowel disease. Am J Physiol Gastrointest Liver Physiol 309, G71-G77. 